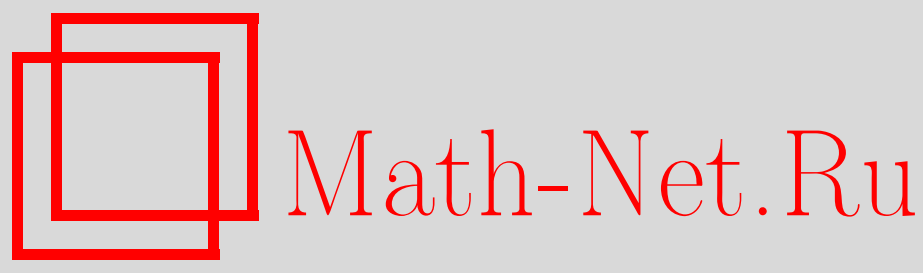

Е. Е. Тареева, В. Н. Рыжов, Закритическая жидкость частиц с потенциалом Юкавы: новое приближение для прямой корреляционной функции и линия Видома, TMФ, 2016, том 189, номер 3, 464-476

DOI: https://doi.org/10.4213/tmf9246

Использование Общероссийского математического портала Math-Net.Ru подразумевает, что вы прочитали и согласны с пользовательским соглашением http://www . mathnet.ru/rus/agreement

Параметры загрузки:

IP : 35.173 .219 .149

26 апреля 2023 г., 12:53:44

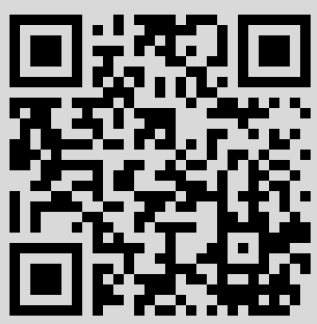


Том 189, № 3

декабрь, 2016

\title{
ЗАКРИТИЧЕСКАЯ ЖИДКОСТЬ ЧАСТИЦ С ПОТЕНЦИАЛОМ ЮКАВЫ: НОВОЕ ПРИБЛИЖЕНИЕ ДЛЯ ПРЯМОЙ КОРРЕЛЯЦИОННОЙ ФУНКЦИИ И ЛИНИЯ ВИДОМА
}

\begin{abstract}
Предложено приближение для прямой корреляционной функции, соответствующее линеаризации по $-\beta \phi(r)$ обобщенного среднесферического приближения для системы твердых сфер, к которым вне твердого ядра добавлено произвольное число потенциалов Юкавы. Полученные результаты применены к исследованию поведения максимумов термодинамических функций отклика в закритической области жидкости с двухчленным потенциалом Юкавы, имитирующим потенциал Леннарда-Джонса.
\end{abstract}

Ключевые слова: прямая корреляционная функция, закритический флюид, линия Видома, потенциал Юкавы.

DOI: $10.4213 / \operatorname{tmf9246}$

\section{1. ВВЕДЕНИЕ}

В последние десятилетия сильно возрос интерес к исследованию поведения сверхкритической жидкости - состояния, находящегося за критической точкой [1]-[7]. Это связано прежде всего с тем, что лишь недавно удалось достичь в эксперименте области температур и давлений, в которой находится сверхфлюид для большинства веществ. Кроме того, оказалось, что сверхкритические флюиды являются очень хорошими растворителями и интересны с точки зрения промышленных технологий в химии и фармакологии [1]. Что касается теоретических аспектов физики закритического флюида, они интересны и сами по себе. Наиболее важными задачами при этом являются описание термодинамического поведения флюида вблизи критической точки и исследование изменения динамики при понижении температуры

Работа поддержана грантами Российского научного фонда № 14-22-00093 (Е. Е. Тареева, главы 2 и 3) и № 14-12-00820 (В. Н. Рыжов, главы 1 и 4).

* Институт физики высоких давлений им. Л.Ф. Верещагина РАН, Троицк, Московская обл., Россия. E-mail: etare@ms2.inr.ac.ru, ryzhov@hppi.troitsk.ru 
(см. обзор [2]). В настоящей статье мы будем иметь дело с первой из указанных задач.

В критической точке $\left(T_{\mathrm{c}}, P_{\mathrm{c}}\right)$ заканчивается линия фазового перехода первого рода жидкость-газ на равновесной фазовой диаграмме в переменных $T, P$. В этой точке расходится величина корреляционной длины $\xi$ [8]. В закритической области свойства флюида меняются непрерывным образом, без сингулярностей, но физические величины, определяемые вторыми производными термодинамического потенциала Гиббса, ведут себя весьма сложно, проходя через максимумы и образуя систему “гребней", сходящихся к критической точке. Такими величинами являются, например, изотермическая сжимаемость $\beta_{T}$, коэффициент теплового расширения $\alpha_{p}$, теплоемкость $c_{p}$. Линия, на которой лежат максимумы корреляционной длины и виртуально должны были бы лежать все указанные максимумы, поскольку все указанные величины связаны с корреляционной длиной, была названа линией Видома ${ }^{1)}$ в работе Стенли с соавторами [11]. На самом деле, как было показано недавно, ее длина, т. е. область, где линии максимумов практически совпадают, составляет доли от $T_{\mathrm{c}}$ и $P_{\mathrm{c}}$. Можно говорить о линии Видома и для перехода жидкость-жидкость. Оба типа линии Видома интенсивно исследуются в последнее время [2], [11]-[30].

Обычно для исследования поведения термодинамических функций отклика в закритической области либо используются методы компьютерного моделирования, либо проводятся расчеты на основе феноменологического или полуфеноменологического уравнения состояния. Как в том, так и в другом случае основная исходная характеристика линии Видома - корреляционная длина - остается за пределами возможностей подхода. Так, в работе [17] для модельной системы с потенциалом Леннарда-Джонса с помощью компьютерного моделирования были найдены положения максимумов флуктуаций плотности, теплоемкости, коэффициента теплового расширения и сжимаемости. Было обнаружено, что полученные линии максиму-

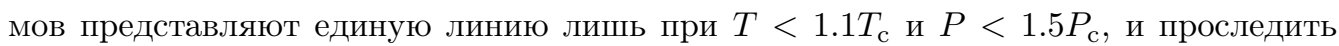

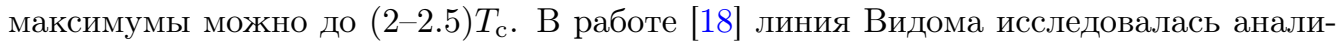
тически, на основе уравнения состояния в модели Ван-дер-Ваальса. Были получены аналитические выражения для положений экстремумов теплоемкости, коэффициента теплового расширения, сжимаемости и флуктуаций плотности. Оказалось, что так же, как в предыдущем случае, линии максимумов сливаются в единую линию

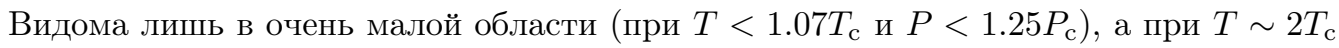
вовсе размываются.

Сугубо микроскопический подход, основанный на использовании прямой корреляционной функции, был предложен в работе [19]. Важно, что в таком подходе можно получить и корреляционную длину. Была рассмотрена закритическая область для системы с потенциалом, представляющим твердые сферы, с добавлением притягивающей прямоугольной ямы. Для прямой корреляционной функции $C(r)$ использовалось простое приближение, широко применяемое в задачах статистической механики и обычно называемое среднесферическим приближением [31]: внутри сферы для $C(r)$ берется известное решение $C_{\mathrm{HS}}$ для системы твердых сфер [32]-[34], а вне сферы полагается $C(r)=-\beta \phi(r)$, где $\beta$ - обратная температура, а $\phi(r)$ - потенциал вне сферы (линейный член приближения Перкуса-Йевика для $C(r))$. Средне-

1) Сам Б. Видом этой линией не занимался, но внес большой вклад в теорию критических явлений (о других линиях см. [9], [10]). 
сферическое приближение было предложено [35], [36] (см. также [37], [38]) для систем частиц с твердым ядром. В работе [19] было показано, что линия максимумов корреляционной длины вписывается в набор максимумов термодинамических функций отклика и весь этот набор очень быстро расширяется, а максимумы размываются. Для всех величин были получены аналитические выражения.

Естественным следующим шагом нам представляется получение аналитических результатов при использовании улучшенных приближений для $C(r)$. В основу приближения, предлагаемого в настоящей работе, положено обобщенное среднесферическое приближение (ОССП) [31], базирующееся на классических работах Бакстера [32], [39]. Поскольку непосредственное использование ОССП приводит к большим техническим трудностям, так что окончательные результаты могут быть представлены лишь численно, мы предлагаем линеаризованное ОССП. Используемая нами в настоящей работе прямая корреляционная функция строится так, чтобы вне радиуса твердой сферы она по-прежнему равнялась - $\beta \phi$, а вся функция в целом удовлетворяла уравнению Орнштейна-Цернике (ОЦ) в линейном по $\beta \phi$ приближении. Нам представляется, что такое приближение является в определенной степени самосогласованным и даже, в некотором смысле, более последовательным, чем ОССП. Существенно, что оно позволяет получить аналитические результаты для рассматриваемого в работе важного случая суммы потенциалов Юкавы, достаточное число членов в которой может приблизить любой потенциал с хорошей степенью точности.

Подробное описание этого приближения и получение общего результата для приближенной прямой корреляционной функции изложено в разделе 2. Раздел 3 посвящен применению полученной прямой корреляционной функции к жидкости с потенциалом, представляющим собой сумму двух потенциалов Юкавы, с параметрами, имитирующими потенциал Леннарда-Джонса. Получены аналитические результаты для положения линий максимумов корреляционной длины, флуктуаций плотности и изотермической сжимаемости в сверхкритической области. Набор линий максимумов расширяется и полностью размывается на небольшом расстоянии от критической точки.

\section{2. ЛИНЕАРИЗОВАННОЕ ОБОБЩЕННОЕ СРЕДНЕСФЕРИЧЕСКОЕ ПРИБЛИЖЕНИЕ}

Как известно, существует точное решение уравнения ОЦ для прямой корреляционной функции системы твердых сфер $C_{\mathrm{HS}}(r)$ при замыкании Перкуса-Йевика (см. [33]), т. е. при условии, что вне радиуса твердой сферы $C(r)=0$. Это решение было получено почти одновременно в работах [32]-[34] и имеет следующий вид (который нам понадобится в дальнейшем):

$$
C_{\mathrm{HS}}(r)=-\lambda_{1}-6 \eta \lambda_{2} r-\frac{1}{2} \eta \lambda_{1} r^{3}
$$

где

$$
\lambda_{1}=\frac{(1+2 \eta)^{2}}{(1-\eta)^{4}}, \quad \lambda_{2}=-\frac{(1+(1 / 2) \eta)^{2}}{(1-\eta)^{4}}
$$

и введена приведенная плотность $\eta=\pi \rho d^{3} / 6(\rho-$ плотность частиц в системе, $d-$ диаметр твердой сферы, в дальнейшем $d=1)$. 
Мы далее следуем работам Бакстера [32], [39] и работам, в которых этот метод применялся к системам с суммой потенциалов Юкавы [40]-[42]. В последующем эти работы использовались разными авторами при рассмотрении различных физических задач (см., например, [43]), причем серьезно продвинуться удалось только в случае одного члена в сумме (см., например, [44]). Настоящая работа наиболее близка по духу работам [42], [44], [47].

Хорошо известное соотношение ОЦ связывает прямую корреляционную функцию $C(r)$ с парной функцией распределения $g(r)$ и в случае однокомпонентной жидкости со сферически-симметричным потенциалом имеет вид

$$
h(r)=C(r)+\rho \int c\left(\left|\vec{r}^{\prime}\right|\right) h\left(\left|\vec{r}-\vec{r}^{\prime}\right|\right) d \vec{r}^{\prime},
$$

где $h(r)=g(r)-1$. В случае потенциалов с твердым ядром (радиус которого мы принимаем равным единице) имеем $h(r)=-1$ при $r<1$. Если при этом добавить какое-нибудь замыкание к уравнению (2), например сделать предположение о виде $C(r)$ при $r>1$, то соотношение ОЦ станет уравнением. В частности, можно положить $C(r)=-\beta \phi(r)$ при $r>1$ (см. [35]). Такое уравнение для $C(r)=0$ при $r>1$ и было решено точно в работах [32]-[34].

Напомним кратко метод Бакстера, которым мы будем пользоваться в дальнейшем. Бакстер [32] показал, что можно получить факторизацию Винера-Хопфа для функции

$$
\tilde{A}(k)=1-\rho \widetilde{C}(k),
$$

где $\widetilde{C}(k)$ - фурье-образ прямой корреляционной функции, т. е. она может быть представлена в виде

$$
\tilde{A}(k)=\widetilde{Q}(k) \widetilde{Q}(-k),
$$

причем функция $1-\widetilde{Q}(k)$ фурье-интегрируема вдоль действительной оси, так что можно определить функцию $Q(r)$ с помощью соотношения

$$
\begin{aligned}
2 \pi \rho Q(r) & =\frac{1}{2 \pi} \int_{-\infty}^{\infty} e^{-i k r}[1-\widetilde{Q}(k)] d k \\
\widetilde{Q}(k) & =1-2 \pi \rho \int_{0}^{R} e^{i k r} Q(r) d r
\end{aligned}
$$

Функция $Q(r)$ обладает следующими свойствами. Это действительная непрерывная функция при $r>0$ и $Q(r)=0$ при $r<0$. Если $C(r)=0$ при $r>R$, то также и $Q(r)=0$ при $r>R$. С помощью этой функции уравнение ОЦ было записано Бакстером в виде двух уравнений:

$$
\begin{aligned}
& r C(r)=-Q^{\prime}(r)+12 \eta \int_{0}^{R} Q^{\prime}(t) Q(t-r) d t \\
& r h(r)=-Q^{\prime}(r)+12 \eta \int_{0}^{R}(r-t) h(|r-t|) Q(t-r) d t .
\end{aligned}
$$

Эти уравнения обладают существенными преимуществами по сравнению с уравнением ОЦ. В частности, они являются одномерными, а не трехмерными. Кроме 
того, в уравнении (4) в случае потенциала с твердым ядром очень удобно использовать условие $h(r)=-1$ при $r<1$. Для системы твердых сфер при условии, что $C(r)=0$ при $r>1$ (т. е. $R=1$ ), из (4) получаем

$$
Q_{\mathrm{HS}}(r)=\frac{1}{2} a_{\mathrm{HS}}\left(r^{2}-1\right)+b_{\mathrm{HS}}(r-1)
$$

где

$$
a_{\mathrm{HS}}=\frac{1+2 \eta}{(1-\eta)^{2}}, \quad b_{\mathrm{HS}}=-\frac{3 \eta}{2(1-\eta)^{2}},
$$

а из (3) получаем выражение (1).

Оказалось, что метод Бакстера может быть успешно применен и к другим потенциалам с твердым ядром, в частности к мультикомпонентному потенциалу Юкавы [40]. Такое приближение, когда уравнение ОЦ решается при замыкании при $r>1$,

$$
C(r)=-\beta \sum_{1}^{n} \frac{\epsilon_{i}}{r} e^{-z_{i}(r-1)},
$$

было названо обобщенным среднесферическим приближением [31]. Это название учитывает тот факт, что почти всякий парный потенциал можно представить суммой потенциалов Юкавы при достаточном числе членов в сумме. В работе [40] был получен общий вид функции $Q(r)$ в этом случае. Уравнения для зависящих от плотности и параметров потенциала коэффициентов, входящих в $Q(r)$, получающиеся после подстановки в (3) и (4) и соответствующего интегрирования, представляют собой $2 n+2$ алгебраических уравнения, из которых только два линейные. За исключением некоторых предельных случаев физические результаты были получены лишь для $n=1$ [44], когда нелинейное уранение является уравнением четвертого порядка.

Мы предлагаем использовать линейное приближение в теории [40], т. е. наша цель - найти линейные по - $\beta \phi(r)$ поправки к $C_{\mathrm{HS}}(r)(r<1)$ такие, чтобы уравнение ОЦ с мультиюкавским замыканием (7) удовлетворялось с этой линейной точностью. Это значит, например, что первое уравнение Бакстера (3) запишется теперь в следующем виде $(r<1)$ :

$$
\begin{aligned}
r C_{\mathrm{HS}}(r)+r \delta C(r)= & -Q_{\mathrm{HS}}^{\prime}(r)-\delta Q^{\prime}(r)+12 \eta \int_{0}^{1} Q_{\mathrm{HS}}^{\prime}(t) Q_{\mathrm{HS}}(t-r) d t+ \\
& +12 \eta \int_{0}^{1} Q_{\mathrm{HS}}^{\prime}(t) \delta Q(t-r) d t+12 \eta \int_{r}^{1+r} \delta Q^{\prime}(t) Q_{\mathrm{HS}}(t-r) d t .
\end{aligned}
$$

Нетрудно показать, следуя [40], что $\delta Q(r)$ имеет вид

$$
\delta Q(r)=\frac{1}{2} a_{1}\left(r^{2}-1\right)+b_{1}(r-1)+\sum_{i=1}^{n} c_{i}\left(e^{-z_{i} r}-e^{-z_{i}}\right)+\sum_{i=1}^{n} d_{i} e^{-z_{i} r}
$$

при $r \leqslant 1$ и

$$
\delta Q(r)=\sum_{i=1}^{n} d_{i} e^{-z_{i} r}
$$

при $r>1$. 
Коэффициенты $a_{1}, b_{1}, c_{i}, d_{i}$ определяются с помощью уравнения (4). При $r<1$ оно принимает вид

$$
\begin{aligned}
-r= & -Q_{\mathrm{HS}}^{\prime}(r)-\delta Q^{\prime}(r)-12 \eta \int_{0}^{1}(r-t) Q(t) d t- \\
& -12 \eta \int_{1}^{r+1}(r-t) \delta Q(t) d t-12 \eta \int_{1+r}^{\infty}(r-t) \delta Q(t) h(|r-t|) d t .
\end{aligned}
$$

Входящую в это уравнение функцию $h(r)$ для $r>1$ можно определить, сделав преобразование Лапласа линеаризованного уравнения (4) для $r>1$ и разрешая полученное уравнение относительно

$$
q(s)=\int_{0}^{\infty} Q(t) e^{-s t} d t
$$

что позволяет сделать уравнение (11) замкнутым. Проведя интегрирования, получим следующую систему $2 n+2$ линейных алгебраических уравнений:

$$
\begin{aligned}
b_{1} & =-\frac{3 \eta}{2} a_{1}-2 \eta b_{1}+12 \eta \sum_{i=1}^{n}\left[c_{i}\left[\frac{1}{z_{i}^{2}}-\left(\frac{1+z_{i}}{z_{i}^{2}}+\frac{1}{2}\right) e^{-z_{i}}\right]+\frac{d_{i}}{z_{i}^{2}}\right], \\
1-a_{1} & =-4 \eta a_{1}-6 \eta b_{1}+12 \eta \sum_{i=1}^{n}\left[c_{i}\left[\frac{1}{z_{i}}-\frac{1+z_{i}}{z_{i}} e^{-z_{i}}\right]+\frac{d_{i}}{z_{i}}\right], \\
K_{i} e^{z_{i}} & =z_{i} d_{i}\left[1-12 \eta q\left(z_{i}\right), \quad i=1, \ldots, n,\right. \\
c_{i}+d_{i} & =12 \eta\left[\sigma\left(z_{i}\right)\left(c_{i}+d_{i}\right)-\tau\left(z_{i}\right) c_{i} e^{-z_{i}}\right], \quad i=1, \ldots, n .
\end{aligned}
$$

Для функции $q(s)$ в нашем приближении достаточно использовать ее выражение для системы твердых сфер:

$$
\begin{aligned}
q(s) & =\sigma(s)-\tau(s) e^{-s} \\
\sigma(s) & =a_{\mathrm{HS}}\left(\frac{1}{s^{3}}-\frac{1}{2 s}\right)+b_{\mathrm{HS}}\left(\frac{1}{s^{2}}-\frac{1}{s}\right), \quad \tau(s)=a_{\mathrm{HS}}\left(\frac{1}{s^{3}}+\frac{1}{s^{2}}\right)+b_{\mathrm{HS}} \frac{1}{s^{2}}
\end{aligned}
$$

Заметим, что каждая из $n$ пар уравнений $(14),(15)$ не зависит от других уравнений, т. е. для каждого $i$ легко получаем решение для $d_{i}, c_{i}$ и затем из $(12),(13)$ - поправки к $a_{\mathrm{HS}}$ и $b_{\mathrm{HS}}$.

\section{3. ЛИНИЯ ВИДОМА В ЗАКРИТИЧЕСКОЙ ОБЛАСТИ ЖИДКОСТИ ЧАСТИЦ С ВЗАИМОДЕЙСТВИЕМ В ВИДЕ СУММЫ ДВУХ ПОТЕНЦИАЛОВ ЮКАВЫ}

Выше мы получили выражение для прямой корреляционной функции системы с потенциалом, являющимся суммой произвольного числа $n$ потенциалов Юкавы. Проведем теперь конкретный расчет для $n=2$.

Пусть потенциал рассматриваемой системы имеет вид

$$
\begin{array}{ll}
\phi(r)=\infty, & r \leqslant 1, \\
\phi(r)=\frac{\epsilon}{r}\left[e^{-z_{1}(r-1)}-e^{-z_{2}(r-1)}\right], & r>1 .
\end{array}
$$


Ниже мы проведем конкретный расчет для значений констант $z_{1}$ и $z_{2}$, которые позволяют имитировать потенциал Леннарда-Джонса (см., например, [48], [49]), а именно, $z_{1}=14.735, z_{2}=2.68$.

Для прямой корреляционной функции мы используем полученные выше результаты, так что она представляется состоящей из трех частей:

$$
\begin{array}{lll}
C(r)=C_{\mathrm{HS}}(r)+\delta C(r), & r \leqslant 1, \\
C(r)=\frac{K_{1}}{r} e^{-z_{1}(r-1)}+\frac{K_{2}}{r} e^{-z_{2}(r-1)}, & r>1,
\end{array}
$$

где $K_{1}=-\beta \epsilon, K_{2}=\beta \epsilon$.

Используя полученные в разделе 2 результаты для $C(r)$ при $r<1$, мы можем теперь написать

$$
\begin{aligned}
-r \delta C(r)= & \lambda_{1}^{\prime} r+6 \eta\left(\lambda_{2}^{\prime}+\lambda_{2}^{\prime \prime}\right) r^{2}+\frac{1}{2} \lambda_{1}^{\prime} r^{4}+ \\
& +\sum_{i=1,2} \mu_{i} \sqrt{z_{i}}\left(1-e^{-z_{i} r}\right)+\sum_{i=1,2} \nu_{i}\left[\cosh \left(z_{i} r\right)-1\right]
\end{aligned}
$$

так что

$$
-\int_{0}^{1} r^{2} \delta C(r) d r=\frac{1}{3} \lambda_{1}^{\prime}+\frac{3}{2} \eta\left(\lambda_{2}^{\prime}+\lambda_{2}^{\prime \prime}\right)+\frac{1}{12} \lambda_{1}^{\prime}+\sum_{i=1,2} \mu_{i} \phi_{1}\left(z_{i}\right)+\sum_{i=1,2} \nu_{i} \phi_{2}\left(z_{i}\right) .
$$

Здесь

$$
\begin{aligned}
& \lambda_{1}^{\prime}=2 \frac{1+2 \eta}{(1-\eta)^{2}} \sum_{i=1,2} K_{i} \frac{e^{z_{i}}}{z_{i}} \frac{1}{V_{i}^{2}(\eta)}\left[72 \eta^{2} A_{i}(\eta)-12 \eta(1+2 \eta) B_{i}(\eta)\right] \\
& \lambda_{2}^{\prime}=-\frac{2+\eta}{(1-\eta)^{2}} \sum_{i=1,2} K_{i} \frac{e^{z_{i}}}{z_{i}} \frac{1}{V_{i}^{2}(\eta)}\left[12 \eta(1+2 \eta) A_{i}(\eta)-6 \eta(2+\eta) B_{i}(\eta)\right] \\
& \lambda_{2}^{\prime \prime}=2(1+2 \eta) \sum_{i=1,2} K_{i} \frac{e^{z_{i}}}{z_{i}} \frac{U_{i}(\eta)}{V_{i}^{2}(\eta)}
\end{aligned}
$$

$A_{i}(\eta) \equiv A\left(z_{i}, \eta\right)$ и т. д., при этом

$$
\begin{aligned}
A(z, \eta) & =\frac{1}{z^{2}}(V(z, \eta)-U(z, \eta))+\left(\frac{1+z}{z^{2}}+\frac{1}{2}\right) e^{-z} U(z, \eta), \\
B(z, \eta) & =\frac{1}{z}(V(z, \eta)-U(z, \eta))+\frac{1+z}{z} e^{-z} U(z, \eta), \\
U(z, \eta) & =1-\frac{2 \eta}{z^{3}}\left(z^{3}-3 z^{2}+6\right)+\frac{\eta^{2}}{z^{3}}\left(z^{3}-6 z^{2}+18 z-24\right), \\
V(z, \eta)-U(z, \eta) & =\frac{e^{-z}}{z^{3}}\left(12 \eta(1+z)+6 \eta^{2}(z+4)\right), \\
\phi_{1}(z) & =\sqrt{z}\left[\frac{1}{2}-\frac{1}{z^{2}}+\frac{z+1}{z^{2}} e^{-z}\right] \\
\phi_{2}(z) & =-\frac{1}{2}+\frac{1}{z^{2}}-\frac{z+1}{2 z^{2}} e^{-z}+\frac{z-1}{2 z^{2}} e^{z},
\end{aligned}
$$




$$
\begin{aligned}
\sum_{i=1,2} \mu_{i} \phi_{1}\left(z_{i}\right)+\nu_{i} \phi_{2}\left(z_{i}\right)= & 24 \eta \sum_{i=1,2} K_{i} \frac{1}{z_{i} V_{i}^{2}}\left[(1+2 \eta) \frac{1+z_{i}}{z_{i}^{3}}-\frac{3 \eta}{2} \frac{1}{z_{i}^{2}}\right] \times \\
& \times\left[V_{i} \phi_{1}\left(z_{i}\right)+\left(V_{i}-U_{i}\right) \phi_{2}\left(z_{i}\right)\right] .
\end{aligned}
$$

Будем использовать далее известную связь (см. [31], [50])

$$
\left(k_{\mathrm{B}} T\right)^{-1}\left(\frac{\partial P}{\partial \rho}\right)_{T}=1-\rho \int C(r) d r=1-\rho \widetilde{C}_{0} .
$$

Ниже мы будем использовать без значка тильды следующие переменные:

$$
\widetilde{P}=\frac{P \pi}{6 \epsilon}, \quad \widetilde{T}=\frac{k_{\mathrm{B}} T}{\epsilon} .
$$

Теперь $K_{1}=-1$ и $K_{2}=1$. Полученная таким образом температура будет в два раза отличаться от температуры в леннард-джонсовских единицах (см. [49]). Вклад в $\partial P / \partial \eta$ от интегрирования по области от нуля до бесконечности равен $-24 \eta \Delta$, где

$$
\Delta=\sum_{i=1,2} K_{i} \frac{1+z_{i}}{z_{i}^{2}}
$$

(в нашем случае $24 \Delta=10.5574$ ).

Используя приведенные выше формулы, запишем

$$
\frac{\partial P}{\partial \eta}=T \frac{(1+2 \eta)^{2}}{(1-\eta)^{4}}-24 \Delta \eta+\frac{\Psi_{1}(\eta)}{(1-\eta)^{2} V_{1}(\eta)^{2}}+\frac{\Psi_{2}(\eta)}{(1-\eta)^{2} V_{2}(\eta)^{2}}
$$

где $\Psi_{1}(\eta)$ и $\Psi_{2}(\eta)$ - полиномы, содержащие от второй до седьмой степени $\eta$.

Решение системы уравнений

$$
\frac{\partial P}{\partial \eta}=0, \quad \frac{\partial^{2} P}{\partial \eta^{2}}=0
$$

дает параметры критической точки:

$$
\eta_{\mathrm{c}}=0.18771, \quad T_{\mathrm{c}}=0.79795
$$

Рассмотрим теперь поведение (которое упоминалось в разделе 1) максимумов некоторых термодинамических величин в закритической области.

С помощью выражения (22) нетрудно получить величину флуктуаций плотности $T(\partial \eta / \partial P)_{T}=\zeta_{T}$. На рис. 1 приведены кривые $\zeta_{T}(\eta)$ при значениях температуры $T=0.85,0.90,0.95,1.0,1.1$ в закритической области. Кривая положения максимумов на изотермах определяется из уравнения

$$
\frac{\partial^{2} P}{\partial \eta^{2}}=0
$$

и приведена на рис. 2. 


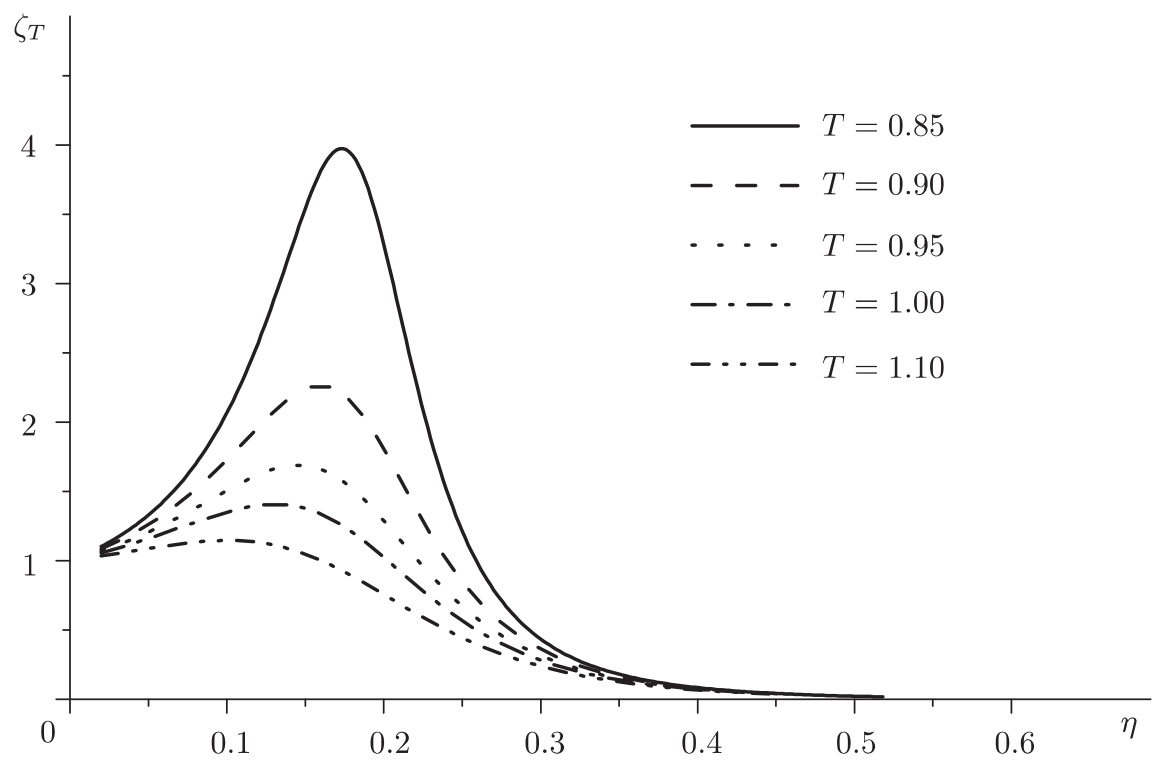

Рис. 1. Поведение флуктуаций плотности $\zeta_{T}$ как функция плотности на изотермах.

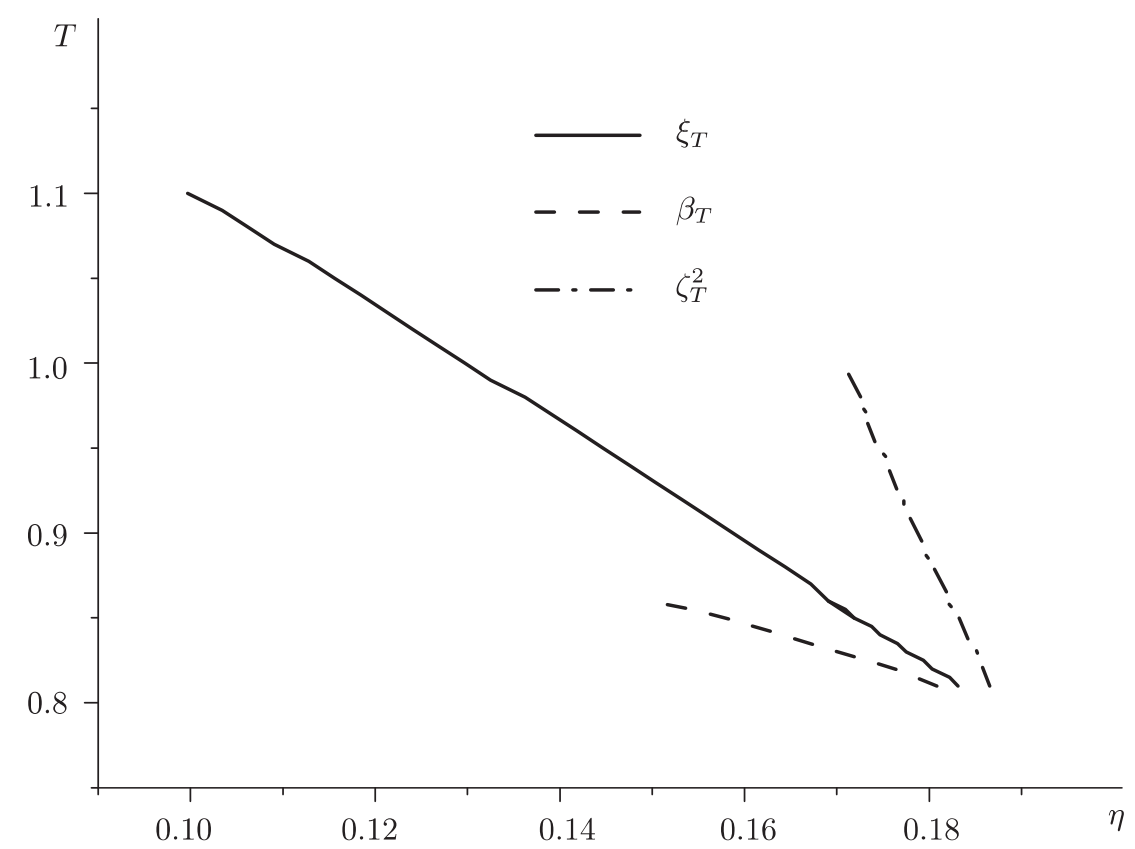

Рис. 2. Линии максимумов флуктуаций плотности $\zeta_{T}$, изотермической сжимаемости $\beta_{T}$ и квадрата корреляционной длины $\xi_{T}^{2}$ на плоскости $(\eta, T)$. 


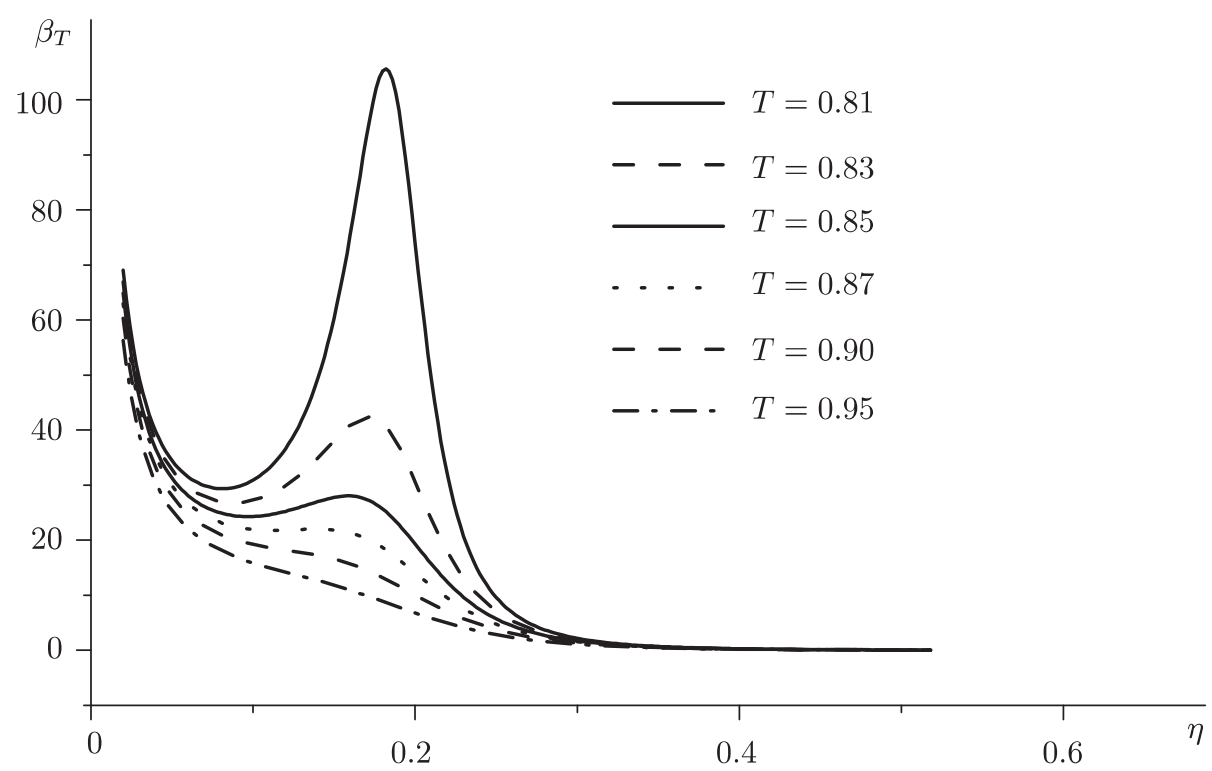

Рис. 3. Изотермическая сжимаемость $\beta_{T}$ как функция плотности на изотермах.

На рис. 3 приведены кривые для изотермической сжимаемости

$$
\beta_{T}=-\frac{1}{V}\left(\frac{\partial V}{\partial P}\right)_{T}=\frac{1}{\eta}\left(\frac{\partial \eta}{\partial P}\right)_{T}
$$

при температурах $T=0.81,0.83,0.85,0.87,0.90,0.95$.

Положение линии максимумов на изотермах описывается решением $T(\eta)$ (рис. 2 ) уравнения

$$
\frac{\partial P}{\partial \eta}+\eta \frac{\partial^{2} P}{\partial \eta^{2}}=0
$$

Кроме того, знание прямой корреляционной функции позволяет получить корреляционную длину $\xi$, используя известные формулы (см. [31], [50]):

$$
\xi^{2}=\frac{R^{2}}{1-\rho \widetilde{C}_{0}(T)}
$$

где

$$
R^{2}=\frac{\rho}{6} \int C(r) r^{2} d r
$$

В нашем случае выражение для $1-\rho \widetilde{C}_{0}$ уже было приведено выше (см. формулы (21), (22)), а для нахождения $R^{2}$ будем снова использовать соотношение (18). Теперь

$$
R^{2}=4 \eta \int_{0}^{1} r^{4} C_{\mathrm{HS}}(r) d r+\frac{4 \eta \Delta^{\prime}}{T}+\frac{4 \eta}{T} \int_{0}^{1} r^{4} \delta C(r) d r
$$

где

$$
\Delta^{\prime}=\sum_{i=1,2} K_{i}\left[\frac{1}{z_{i}}+\frac{3}{z_{i}^{2}}+\frac{6}{z_{i}^{3}}+\frac{6}{z_{i}^{4}}\right]
$$




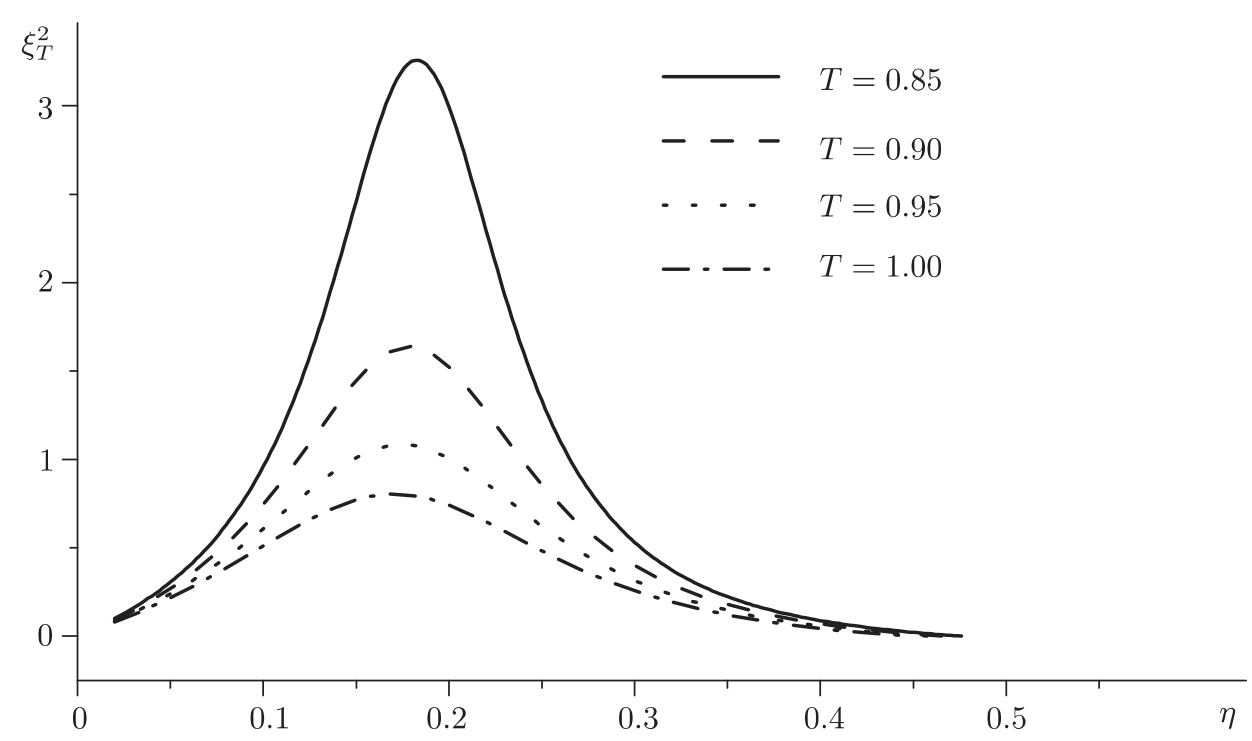

Рис. 4. Квадрат корреляционной длины $\xi_{T}^{2}$ как функция плотности на изотермах.

(в нашем случае $\left.\Delta^{\prime}=1.135\right)$. Кроме того,

$$
-\int_{0}^{1} r^{4} \delta C(r) d r=\frac{1}{5} \lambda_{1}^{\prime}+\eta\left(\lambda_{2}^{\prime}+\lambda_{2}^{\prime \prime}\right)+\frac{\eta}{16} \lambda_{1}^{\prime}+\sum_{i=1,2} \mu_{i} \chi_{1}\left(z_{i}\right)+\sum_{i=1,2} \nu_{i} \chi_{2}\left(z_{i}\right),
$$

где

$$
\begin{aligned}
& \chi_{1}(z)=\sqrt{z}\left[\frac{1}{4}-\frac{6}{z^{4}}+e^{-z}\left(\frac{1}{z}+\frac{3}{z^{2}}+\frac{6}{z^{3}}+\frac{6}{z^{4}}\right)\right] \\
& \chi_{2}(z)=-\frac{1}{4}+\frac{6}{z^{4}}+\frac{e^{z}}{2}\left(\frac{1}{z}-\frac{3}{z^{2}}+\frac{6}{z^{3}}-\frac{6}{z^{4}}\right)-\frac{e^{-z}}{2}\left(\frac{1}{z}+\frac{3}{z^{2}}+\frac{6}{z^{3}}+\frac{6}{z^{4}}\right)
\end{aligned}
$$

так что

$$
R^{2}=\frac{\eta}{20} \frac{-16+11 \eta-4 \eta^{2}}{(1-\eta)^{4}}+\frac{4 \eta \Delta^{\prime}}{T}+\frac{1}{T}\left[\frac{\Psi_{3}(\eta)}{(1-\eta)^{2} V_{1}(\eta)^{2}}+\frac{\Psi_{4}(\eta)}{(1-\eta)^{2} V_{2}(\eta)^{2}}\right] .
$$

Здесь $\Psi_{3}(\eta)$ и $\Psi_{4}(\eta)$ - снова полиномы, содержащие члены от второй до седьмой степени $\eta$.

На рис. 4 представлен ход квадрата корреляционной длины как функции $\eta$ при температурах $T=0.85,0.90,0.95,1.0$, а на рис. 2 - линия максимумов $\xi^{2}$.

\section{4. ЗАКЛЮЧЕНИЕ}

В статье предложено линеаризованное ОССП для модели с потенциалом твердых сфер, к которому прибавлено произвольное число потенциалов Юкавы. В рамках данного приближения удалось получить аналитическое выражение для прямой корреляционной функции $C(r)$, которое позволяет построить полную термодинамику рассматриваемой системы. Для решения задачи был использован подход, аналогичный развитому ранее Бакстером для точного решения модели твердых сфер. 
Полученное решение для случая двухчленного потенциала Юкавы, имитирующего потенциал Леннарда-Джонса, позволило построить аналитическую модель, подобную модели Ван-дер-Ваальса. В рамках данной модели нами была вычислена критическая точка и рассмотрено поведение корреляционной длины $\xi_{T}$, изотермических флуктуаций плотности $\zeta_{T}$ и изотермической сжимаемости $\beta_{T}$ в закритической области. Оказалось, что в соответствии с нашими предыдущими результатами для потенциала Леннарда-Джонса [17], потенциала с прямоугольной ямой [19], а также модели Ван-дер-Ваальса [18] линии максимумов данных величин сливаются в одну линию (называемую иногда линией Видома) только в самой непосредственной окрестности критической точки. Как уже отмечалось в наших предыдущих публикациях, это позволяет сделать заключение о том, что линия Видома является плохо определенным понятием и не может быть использована для разделения закритической области на жидкостноподобную и газоподобную области. Для этой цели была предложена линия Френкеля [2]-[7], [26]-[29], которая определяет кроссовер между двумя типами динамического поведения сверхкритических флюидов .

Следует отметить, что порядок следования линий максимумов корреляционной длины $\xi_{T}$, изотермических флуктуаций плотности $\zeta_{T}$ и изотермической сжимаемости $\beta_{T}$ в закритической области (см. рис. 2) совпадает с соответствующими результатами, полученными ранее [17]-[19], [30]. Это позволяет заключить, что порядок следования линий максимумов термодинамических восприимчивостей является универсальным и не зависит от конкретной модели.

Благодарности. Авторы благодарны В. В. Бражкину, Ю. Д. Фомину и Е. Н. Циок за полезные обсуждения.

\section{Список литературы}

[1] E. Kiran, P. G. Debenedetti, C. J. Peters (eds.), Supercritical Fluids. Fundamentals and Applications, Kluwer, Dordrecht, 2000.

[2] В. В. Бражкин, А. Г. Ляпин, В.Н. Рыжов, К. Траченко, Ю. Д. Фомин, Е. Н. Циок, УФН, 182:11 (2012), 1137.

[3] В. В. Бражкин, А.Г. Ляпин, В.Н. Рыжов, К. Траченко, Ю. Д. Фомин, Е. Н. Циок, Сверхкритические флюиды: теория и практика, 2014, № 2, 40-50.

[4] V. V. Brazhkin, Yu. D. Fomin, A. G. Lyapin, V. N. Ryzhov, K. Trachenko, Phys. Rev. E, 85:3 (2012), 031203, 12 pp.

[5] V.V. Brazhkin, Yu.D. Fomin, A. G. Lyapin, V.N. Ryzhov, K. Trachenko, Пuсъмa в ЖКЭТФ, 95:3 (2012), 179-184.

[6] V. V. Brazhkin, Yu. D. Fomin, A. G. Lyapin, V. N. Ryzhov, E. N. Tsiok, K. Trachenko, Phys. Rev. Lett., 111:14 (2013), 145901, 5 pp.

[7] D. Bolmatov, V. V. Brazhkin, Yu. D. Fomin, V. N. Ryzhov, K. Trachenko, J. Chem. Phys., 139:23 (2013), 234501.

[8] Г. Стенли, Фазовые переходы и критические явления, Мир, М., 1973.

[9] M. E. Fisher, B. Widom, J. Chem. Phys., 50:9 (1969), 3756-3771.

[10] B. Widom, J.S. Rowlinson, J. Chem. Phys., 52:4 (1970), 1670-1683.

[11] L. Xu, P. Kumar, S. V. Buldyrev, S.-H. Chen, P. H. Poole, E. Sciortino, H. E. Stanley, Proc. Natl. Acad. Sci. USA, 102:48 (2005), 16558-16562.

[12] P. H. Poole, S. R. Becker, F. Sciortino, F. W. Starr, J. Phys. Chem. B, 115:48 (2011), 14176-14183.

[13] G. Franzese, H. E. Stanley, J. Phys.: Condens. Matter, 19:20 (2007), 205126, 16 pp. 
[14] P. F. McMillan, H. E. Stanley, Nature Phys., 6 (2010), 479-480.

[15] G. G. Simeoni, T. Bryk, F. A. Gorelli, M. Krisch, G. Ruocco, M. Santoro, T. Scopigno, Nature Phys., 6 (2010), 503-507.

[16] F. A. Gorelli, T. Bryk, M. Krisch, G. Ruocco, M. Santoro, T. Scopigno, Sci. Rep., 3 (2013), 1203, 5 pp.

[17] V. V. Brazhkin, Yu. D. Fomin, A. G. Lyapin, V. N. Ryzhov, E. N. Tsiok, J. Phys. Chem. B, 115:48 (2011), 14112-14115.

[18] V.V. Brazhkin, V.N. Ryzhov, J. Chem. Phys., 135:8 (2011), 084503, 5 pp., arXiv: 1104.2973.

[19] V.V. Brazhkin, Yu. D. Fomin, V. N. Ryzhov, E. E. Tareyeva, E. N. Tziok, Phys. Rev. E, 89:4 (2014), 042136, 6 pp.

[20] P. Gallo, D. Corradini, M. Rovere, J. Chem. Phys., 139:20 (2013), 204503, 7 pp.

[21] G. Ruppeiner, A. Sahay, T. Sarkar, G. Sengupta, Phys. Rev. E, 86:5 (2012), 052103, 4 pp.

[22] P. Gallo, M. Rovere, J. Chem. Phys., 137:16 (2012), 164503, 6 pp.

[23] D. A. Fuentevilla, M. A. Anisimov, Phys. Rev. Lett., 97:19 (2006), 195702, 4 pp.

[24] P. Gallo, D. Corradini, M. Rovere, Nature Commun., 5 (2014), 5806, 6 pp.

[25] J. L. F. Abascal, C. Vega, J. Chem. Phys., 133:23 (2010), 234502, 8 pp.

[26] Yu. D. Fomin, V. N. Ryzhov, E. N. Tsiok, V. V. Brazhkin, Phys. Rev. E, 91:2 (2015), 022111, 5 pp.

[27] Y.D. Fomin, V. N. Ryzhov, E. N. Tsiok, V. V. Brazhkin, K. Trachenko, Sci. Rep., 4 (2014), 07194, 5 pp.

[28] Yu. D. Fomin, V. N. Ryzhov, E. N. Tsiok, V. V. Brazhkin, Sci. Rep., 5 (2015), 14234, 6 pp.

[29] Yu. D. Fomin, E. N. Tsiok, V. N. Ryzhov, Phys. Rev. E, 87:4 (2013), 042122, 5 pp.

[30] H.-O. May, P. Mausbach, Phys. Rev. E, 85:3 (2012), 031201, 9 pp.

[31] J. P. Hansen, I. R. McDonald, Theory of Simple Liquids, Academic, New York, 1986.

[32] R. J. Baxter, Austral. J. Phys., 21:5 (1968), 563-570.

[33] M. S. Wertheim, Phys. Rev. Lett., 10 (1963), 321-323; J. Math. Phys., 5:5 (1964), 643-651.

[34] E. Thiele, J. Chem. Phys., 39 (1963), 474-479.

[35] J. K. Percus, G. J. Yevick, Phys. Rev., 136:1B (1964), 290-296; J. L. Lebowitz, J. K. Percus, Phys. Rev., 144:1 (1966), 251-258.

[36] R. Lovett, J. Chem. Phys., 66:3 (1977), 1225-1230.

[37] В. Н. Рыжов, Е. Е. Тареева, Ю. Д. Фомин, ТМФ, 167:2 (2011), 284-294.

[38] E. S. Chumakov, Y.D. Fomin, E. L. Shangina, E. E. Tareyeva, E. N. Tsiok, V. N. Ryzhov, Phys. A, 432 (2015), 279-286.

[39] R. J. Baxter, J. Chem. Phys., 52:9 (1970), 4559-4562.

[40] J. S. Høye, L. Blum, J. Statist. Phys., 16:5 (1977), 399-413.

[41] L. Blum, J. S. Høye, J. Statist. Phys., 19:4 (1978), 317-324.

[42] D. Henderson, L. Blum, J. P. Noworyta, J. Chem. Phys., 102:12 (1995), 4973-4975.

[43] Y. Liu, W.-R. Chen, S.-H. Chen, J. Chem. Phys., 122:4 (2005), 044507.

[44] J. H. Herrera, H. Ruiz-Estrada, L. Blum, J. Chem. Phys., 104:16 (1996), 6327-6329.

[45] M. Chen, J. Math. Phys., 27:7 (1985), 1852-1858.

[46] S. Hlushak, A. Trokhymchuk, S. Sokolowski, J. Chem. Phys., 130 (2009), 234511, 10 pp.

[47] M. Ginoza, Mol. Phys., 71:1 (1990), 145-156; D.-M. Duh, L. Mier-Y.-Terán, 90:3 (1997), 373-380; M. Ginoza, J. Phys.: Condens. Matter, 6:8 (1994), 1439-1448.

[48] C.F. Tejero, A. Daanoun, H. N. W. Lakkerkerker, M. Baus, Phys. Rev. E, 51:1 (1995), $558-566$.

[49] M. Bahaa Khedr, S. M. Osman, M. S. Al Busaidi, Phys. Chem. Liq., 47:3 (2009), 237-249.

[50] Р. Балеску, Равновесная и неравновесная статистическая механика, Мир, М., 1978. 